\title{
Large-Scale Fading Coefficients Classification-Aided Power Control Strategy for Mitigating the Pilot Contamination in Massive MIMO Systems
}

\author{
Abdelfettah BELHABIB *, Jamal AMADID, Abdelouhab Zeroual \\ Instrumentation, Signals and Physical Systems (I2SP) Group, Faculty of Sciences Semlalia, Cadi Ayyad University Marrakesh, Morocco
}

\begin{abstract}
Deploying base stations with a massive number of antennas give birth to massive multi-input multi-output technology, which can boost the quality of the wireless communication; however, the limitations imposed by the length of the coherence interval has, directly, limited the number of the orthogonal pilot sequences (OPSs) that can be exploited for acquiring such accurate channel state information at each base station (BS). Hence, the available pilot resources are reused among several cells, which causes the problem of pilot contamination (PC). In this paper, the PC problem is analyzed, and by exploiting the large-scale fading coefficients of the users' equipment (UEs), we have classified the users within each cell; therefore, the same OPS is, solely, reused by the far-distant UEs; hence, the influence of the PC problem is made as small as possible. Since the UEs are subject to different channel conditions, we have consolidated our proposal by a power control algorithm, which boosts the performance of our proposal. Simulation results prove the effectiveness of our proposed decontaminating strategy.
\end{abstract}

Keywords Massive multi-input muli-output technology, Multi-cellular Systems, Pilot Contamination, large-scale fading coefficients, $5 \mathrm{G}$, power control.

AMS 2010 subject classifications 91A28, 60G35, 90B18

DOI: $10.19139 /$ soic-2310-5070-1201

\section{Introduction}

Massive multiple-input multiple-output MIMO (M-MIMO) or Large scale MIMO technology has received considerable attention from academic researchers, which are interested in boosting the quality and reliability of the wireless communication systems, especially, to meet the unprecedented demands for high data rate [1]. To increase the number of degrees-of-freedom of the base stations (BSs), M-MIMO proposes to evolve the BSs with a massive number of antennas, so they can serve, simultaneously, dozens of the users' equipment (UEs) [2]. Indeed, M-MIMO technology faces a serious problem of inter-cell interference (ICI), which is well-known as the problem of pilot contamination (PC) [3]. Globally, the wireless communication between the UEs and their supported BSs is performed in coherent blocks (CBs). Each CB is composed of two rooms, one for channel training, while the second room is reserved for data transmission [4]. Since M-MIMO is operating under TDD mode, the CBs are limited; hence, the room reserved for channel training is also limited, which leads to limit the number of the available pilot sequences that can be exploited for the channel training phase. Consequently, reusing the same pilot sequences within cells is unavoidable, which leads to the problem of PC (Fig. 1). In a nutshell, M-MIMO roughly depends on spatial multiplexing, which in turn depends on the accuracy of the estimated channels at the BSs, and the quality of these channels is degraded because of the PC problem; hence, it is very interesting to overcome the PC problem, so that we can exploit the full advantages of M-MIMO technology.

\footnotetext{
${ }^{*}$ Correspondence to: Abdelfettah BELHABIB (Email: abdelfettah.belhabib@edu.uca.ac.ma). Instrumentation, Signals and Physical Systems (I2SP) Group, Faculty of Sciences Semlalia, Cadi Ayyad University Marrakesh, Morocco
}

ISSN 2310-5070 (online) ISSN 2311-004X (print)

Copyright (C) 2022 International Academic Press 


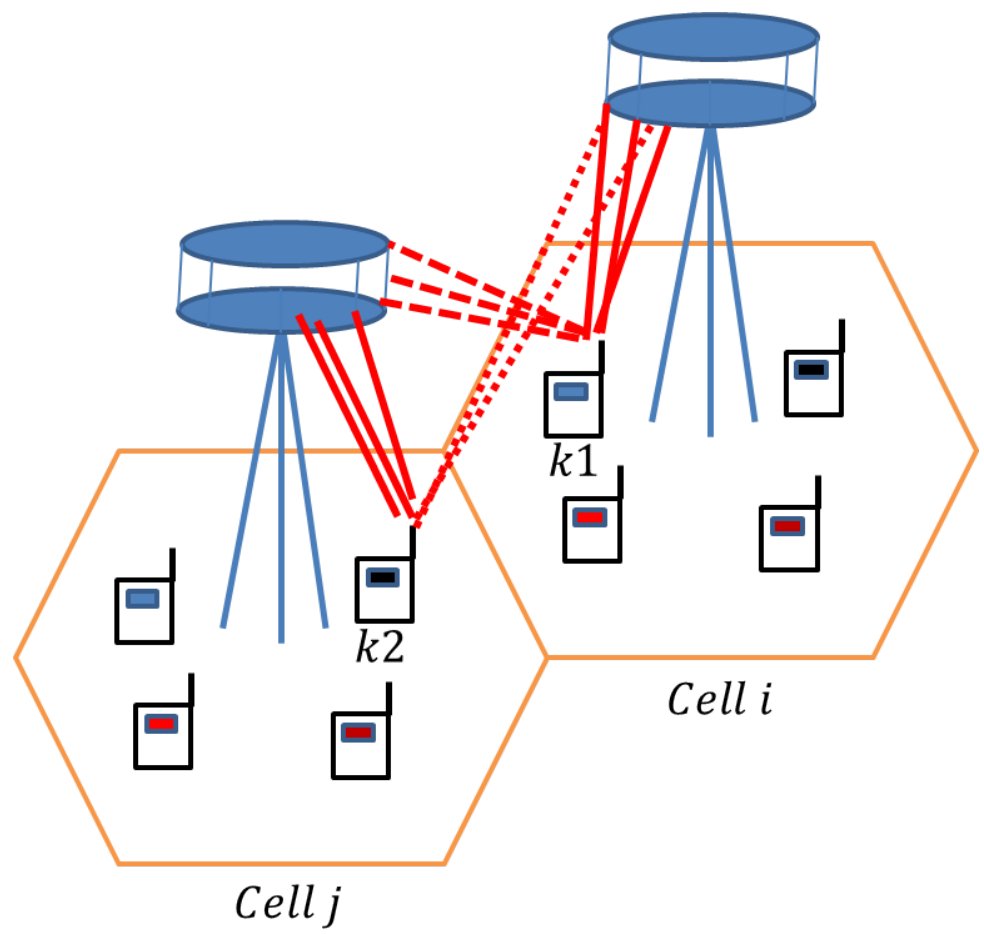

Figure 1. Illustration of the PC problem where the dashed lines represents the PC problem between two UEs $k_{1}$ and $k_{2}$ that reuse the same OPSs.

\subsection{Organization}

The remaining of this paper is as follows. Section 2presents the related works, while the contributions of this paper are presented in Section 2.1. Section 3 presents the adopted system model. The communication schemes are provided in Section 4, while Section 5 gives a short overview of the PC problem. The proposed strategy is presented in section 6. Simulation results are presented in section 7. Finally, a conclusion of the present paper is given in section 8 and section 9 provides an appendix.

\section{Related Works}

The large benefits of M-MIMO, in term of quality-of-services, has triggered the researchers' curiosity to look for new strategies that can alleviate or reduce the sever influence of the PC problem. Thus, for mitigating this problem, different approaches were conducted; the pilot reuse factor is considered in [5], which suggests allocating orthogonal band of frequencies to the adjacent cells; however, the scarcity of the band of frequencies makes this strategy impractical, especially, in such crowded scenarios. The power control based approach is conducted in the work of [6] where the transmit pilot and data power, the length of the $\mathrm{CB}$ is jointly optimized to maximize the achievable rate; however, this study is limited to the uplink phase with no focus on the influence of the PC problem. A power allocation based optimization strategy was proposed in the work of [7], which aims to enhance the energy efficiency of M-MIMO technology under the constraint of imperfect channel state information (CSI); indeed, only a single-cell scenario is considered where the pilot sequences of the UEs are considered to be orthogonal, which is a bit ambiguous since the PC problem is completely ignored. To minimize the transmit power while maintaining a good signal-to-noise-plus-interference ratio (SINR) of the UEs, [8] has proposed a coordinated strategy, which aims to find the optimal beamformer through optimizing the transmit downlink powers, which are constrained by a fixed downlink SINR requirement for the UEs; however, the UEs are subject to different channel conditioning; 
hence, this proposal is vulnerable to diverge during the optimization stage. A power optimization scheme for a multi-UE multi-cell system is provided in [9], which aims to jointly minimize the transmit powers in both uplink and downlink phases. This to find the optimal beam-former while maintaining a fixed SINR target. A coordinated power optimization strategy is proposed in [10], where the BSs coordinate through a backhaul to find an optimal beamformer; this under the assumption that each BSs knows the overall channel vectors, which is practically questionable due to the PC problem and backhaul signaling overhead.

The authors of [11] propose to exploit the UEs' large-scale fading coefficients ( $L S F^{\text {coefficients }}$ ) in designing both precoder and decoders at each BS; however, this proposal is not without repercussions, and it faces the problem of pilot overhead, which can lead to shrink the bandwidth left for data transmission, which affect, badly, the system throughput [12]. The $L S F^{\text {coefficients }}$ were exploited in [13] to construct an optimal precoder that leads to boost the quality of services. A graph of interference was designed in the work of [14], where the LSF $F^{\text {coefficients }}$ are exploited to compute the severity of the PC problem upon each UE, hence, the available orthogonal pilot sequences (OPSs) are optimally assigned to the UEs, where the same OPSs is, solely, allocated to the UEs that can cause to each other the minimum influence of the PC problem. The authors of [15] claims that the PC problem can be alleviated through delaying the training phase of the adjacent cells; on other words, the adjacent cells are not allowed to, synchronously, performing training phase; however, the estimated channels become contaminated by the data symbols, which are transmitted in the adjacent cells. In [16], the authors suggest to alleviate the PC problem through performing the channel training in both uplink and downlink phase, where the UEs are charged to exchange information with their supported BSs before starting data transmission; indeed, this proposal suffers from a high computational complexity. By exploiting the UEs' achievable rate, [17] proposed to, optimally, alloacte the available OPSs to the UEs through minimizing a target function that accounts for the strength of the PC problem upon the UEs. The uplink and the downlink phases were jointly optimized in [18], which helped to enhance the performance of M-MIMO. In [19], the authors proposed to design an optimal multi-cell precoder through the minimization of the undesired signals, significant improvements were achieved; however, the need for manipulating a nonlinear equations escalates the complexity of this strategy. Since the UEs are subject to different channel conditions, the authors of [20] separates the UEs into two groups i.e center and outer UEs; therefore, the same set of the OPSs is reused for the center UEs of the overall cells, while a specific OPSs is allocated to each outer UE; however, this approach requires extra OPSs. Similar to [20], in [21], the UEs were divided into different groups based on their geographical positions, where OPSs are allocated to the UEs that belong to closer sectors; however, intra-sector interference is ignored, furthermore, the UEs are assumed to have such non-overlapping angle-of-arrivals (AoAs). Based on the UEs' LSFcoef ficient, [22] computes the severity of the PC upon each UEs; therefore, the assignment of the available OPSs is controlled by the maximization of the minimum SINR.

\subsection{Contribution}

Since the UEs within cells are subject to different channel conditioning, it is unfair to be treated similarly. Accordingly, we propose to classify the UEs within cells based on their $L S F^{\text {coef ficient }}$; therefore, we allocate, for each UE, a specific OPSs that can help to minimizing the influence of the PC problem. Furthermore, to guarantee some fairness between the UEs; a power control algorithm is employed. Specifically, the prime contributions of this paper are as follows:

- There is no doubt that the UEs that are close to the BSs enjoy with a high QoSs compared to the UEs which are far from BSs; hence, we exploit the $L S F^{\text {coef ficients }}$ to classify the UEs within cells; therefore, OPSs are allocated to the UEs under the constraint that the same OPSs is reused for the UEs that are far from each other.

- Once the UEs are allocated with their specific OPSs, we allocate high powers for the UEs which are far from the BSs, while the UEs that are close to the BSs are allowed to transmit using a -relatively- small power during both pilot and data transmission.

- Instead of sharing information between BSs, which lead to the problem of backhaul signaling, the proposed algorithm requires only to share the UEs' $L S F^{\text {coef ficients }}$ with a central hub, which is connected to all BSs (i.e one network hub connected to a set of seven cells). 


\section{System Model}

Let us consider a typical multi-cell system of $\mathrm{L}$ hexagonal cells. Each cell supports a set of a $K<<M$ randomlydistributed single-antennas UEs, which are served by a centered BS of M antennas. By adopting the Rayleighfading model, the channel vector $g_{j, i, k} \in C^{M \times 1}$ that links a UE "k" of cell "i" to a BS of cell "j" can be expressed by the following product:

$$
g_{j, i, k}=h_{j, i, k} \sqrt{\beta_{j, i, k}},
$$

where $\beta_{j, i, k}$ denotes the $L S F^{\text {coef ficient }}$ and it modulates the path loss and shadow fading, and it is frequency independent, whereas $h_{j, i, k}$ represents the small-scale fading vector, which has a complex Gaussian distribution of zero-mean and variance unity, and it is independent and identically distributed for the K UEs of each cell i.e., $h_{j, i, k} \sim \mathcal{C} N\left(\mathbf{0}, \mathbf{I}_{M}\right)$

\subsection{Law of Large Numbers}

Increasing the number of the antennas at the BSs can leads to makes the channel vectors of the UEs closer to be orthogonal, which means that both the uncorrelated and noisy component vanishes. This result can be explained by the law of large number. Let $\left(g_{j, i, k 1}, g_{j, i, k 2}\right) \in \mathcal{C}^{M \times 1}$ be two independent vectors where $k 1 \neq k 2$ and $g_{j, i, k 1} \sim \mathcal{C N}\left(0, \beta_{j, i, k 1} I_{M}\right), g_{j, i, k 2} \sim \mathcal{C N}\left(0, \beta_{j, i, k 2} I_{M}\right)$. Therefore the law of large numbers can be expressed as

$$
\lim _{M \rightarrow \infty} \frac{g_{j, i, k 1}^{H} g_{j, i, k 1}}{M} \rightarrow \beta_{j, i, k 1} \quad \text { and } \quad \lim _{M \rightarrow \infty} \frac{g_{j, i, k 1}^{H} g_{j, i, k 2}}{M} \rightarrow 0
$$

Based on (2), both the independent channels and the noisy vectors tend to vanish in the asymptotic regime where $M \rightarrow \infty$.

\section{Communication schemes}

The transmission of data symbols between BSs and UEs is preceded by the pilot transmission phase. Thus, by adopting the block-fading channel model, the UEs are obliged to uplink their pilot sequences, which are then exploited at the BSs' side for the estimation of the CSIs. Hence, it can be deduced that any wireless communication -based pilot assignment- is composed of the following steps: pilot sounding for channel training, then data transmission.

To simplify the analysis, we will focus our analyzes on the $k^{\text {th }}$ UE that aims to communicate with the BS of cell 'j' (i.e,. BS-j). First, the $k^{t h}$ UE uplink his pilot sequences $\phi^{[k]} \in C^{1 \times \tau}$, and the received pilot signal $y_{j, k}^{p} \in \mathcal{C}^{M \times \tau}$ at the BS of cell $j$ can be expressed as

$$
y_{j, k}^{p}=\sqrt{\rho_{p}^{k}} \sum_{i=1}^{L} g_{j, i, k} \phi^{[k]}+n_{j}^{p}=\sqrt{\rho_{p}^{k}} g_{j, j, k} \phi^{[k]}+\sqrt{\rho_{p}^{k}} \sum_{i \neq j}^{L} g_{j, i, k} \phi^{[k]}+n_{j}^{p},
$$

where $\rho_{p}^{k}$ represents the power used by the $k^{t h}$ UE to uplink his pilot sequences, while $n_{j}^{p}$ denotes the additive white Gaussian noise (AWGN) at the antennas of the $j^{\text {th }}$ BS.

Therefore, BS-j correlates $y_{j, k}^{p}$ with $\phi^{[k]}$ for estimating the CSI as follows

$$
\hat{g}_{j, j, k}=\frac{y_{j, k}^{p}\left(\phi^{[k]}\right)^{H}}{\sqrt{\rho_{p}^{k}} \tau}=g_{j, j, k}+\sum_{i \neq j}^{L} g_{j, i, k}+\frac{n_{j}^{p}\left(\phi^{[k]}\right)^{H}}{\sqrt{\rho_{p}^{k}}},
$$

where $\phi^{[k]}\left(\phi^{\left[k^{\prime}\right]}\right)^{H}=\tau \delta\left\{k-k^{\prime}\right\}$. From (3), we notice that the estimated channel is a summation of the desired channel $g_{j, j, k}$, the contaminating channels of the UE that reuse the same pilot sequences as the UE ' $\mathrm{k}$ ' i.e, 
$\sum_{i \neq j}^{L} g_{j, i, k}$, and the noisy components $\frac{n_{j}^{p}\left(\phi^{[k]}\right)^{H}}{\sqrt{\rho_{p}^{k}}}$, which can be canceled in the asymptotic regime (i.e,. $M \rightarrow \infty$.

After estimating the CSI $\hat{g}_{j, j, k}$, the BS-j uses this channel for constructing both detector and precoder, respectively, for data detection in the uplink phase, and transmitting data symbols in the downlink phase. In the uplink phase, the received data signal $y_{j, k}^{u}$ at the BS-j can expressed as

$$
y_{j, k}^{u}=\sqrt{\rho_{u}^{k}} \sum_{i=1}^{L} g_{j, i, k} s_{i}^{k}+n_{j}^{u}
$$

where $\rho_{u}^{k}$ represents the power used for transmitting the data symbol $s_{i}^{k}$, while $n_{j}^{u}$ refers to the AWGN, of zeromean and variance unity, at the antennas of BS-j. Therefore, by adopting the matched-filter detector, the BS-j can obtain the following SINR

$$
S I N R_{k, j}^{u}=\frac{\left|g_{j, j, k}^{H} g_{j, j, k}\right|^{2}}{\sum_{i \neq j}^{L}\left|g_{j, i, k}^{H} g_{j, j, k}\right|^{2}+\left|n_{j}^{u} \hat{g}_{j, j, k}^{H}\right|^{2} / \rho_{u}^{k}+b_{j}^{k}}
$$

where the data symbols are considered to have a zero-mean and variance unity, and $b_{j}^{k}$ denotes the uncorrelated components.

For the downlink phase, BS-j adopt the maximum-ratio transmitting to beamforming data symbols towards the UEs. Thus, a simple analysis yields the following downlink SINR

$$
\operatorname{SIN}_{k, j}^{d}=\frac{\left|g_{j, j, k}^{H} g_{j, j, k}\right|^{2}}{\sum_{j \neq i}^{L}\left|g_{i, j, k}^{H} g_{i, j, k}\right|^{2}+\left|n_{j}^{u} \hat{g}_{j, j, k}^{H}\right|^{2} / \rho_{u}^{k}+j_{j}^{k}}
$$

where the $j_{j}^{k}$ denotes the uncorrelated components, which vanish in the asymptotic regime. For more details about the derivation of (5) and (6), we invite the interseted readers to [25].

\section{The influence of the $\mathrm{PC}$ problem}

The scarcity of the OPSs makes the frequency-division duplex useless for M-MIMO systems; hence, TDD is more suitable for M-MIMO since the number of the OPSs required for channel training is solely depend on the number of the UEs. Since the number of the UEs that need to be served is much higher than the available OPSs, it is mandatory to reuse the same OPSs for several UEs that belong to different cells, which in fact lead to the PC problem. This problem bounds the scalability of M-MIMO technology. Mathematically, let call the two equations (5) and (6) and compute their values in the asymptotic regime as follows

$$
\begin{aligned}
& \operatorname{SINR}_{k, j}^{u, M \rightarrow \infty} \rightarrow \frac{\beta_{j, j, k}^{2}}{\sum_{j \neq i}^{L} \beta_{j, i, k}^{2}+1 / \rho_{u}^{k}} \\
& \operatorname{SINR}_{k, j}^{d, M \rightarrow \infty} \rightarrow \frac{\beta_{j, j, k}^{2}}{\sum_{j \neq i}^{L} \beta_{i, j, k}^{2}+1 / \rho_{d}^{k}}
\end{aligned}
$$

From the two equations (7) and (8), it is well seen that their denominators do not vanish in the asymptotic regime, which faces the the improvement of the fulfilled SINR in both uplink and downlink phases. This effect influence also the fulfilled average uplink and downlink achievable rate, which can be expressed, respectively, as[23]

$$
\begin{aligned}
& C_{j, k}^{u}=(1-\mu) E\left\{\log _{2}\left(1+S I N R_{k, j}^{u, M \rightarrow \infty}\right)\right\} \\
& C_{j, k}^{d}=(1-\mu) E\left\{\log _{2}\left(1+S I N R_{k, j}^{d, M \rightarrow \infty}\right)\right\}
\end{aligned}
$$

where $0<\mu<1$ is a parameter that accounts for the loss of spectral efficiency (SE) caused by problem of the pilot overhead. Regarding the fact that average uplink and downlink achievable rate ((9)(10)) depend on the SINR, the fulfilled average uplink/downlink achievable rate is also bounded by the PC problem. 


\section{The Proposed Algorithm}

The proposed strategy can be divided into two folds: UEs classification with pilot assignment and the power control. Accordingly, the two folds are adopted in the following two subsections.

\subsection{UEs' classification and pilot pilot assignment}

To classify the UEs within cells, it is required to know the distance between each UEs and its supported BS. That why we will exploit the UEs' $L S F^{\text {coef ficients }}$, which can be expressed for the $k^{\text {th }}$ UE of cell 'i' to BS-j as

$$
\beta_{j, i, k}=\frac{z_{j, i, k}}{\left(r_{j, i, k} / R\right)^{\alpha}}
$$

where $z_{j, i, k}$ is a log-normal distribution, and it follows a Gaussian distribution of a zero-mean and having a standard deviation $\sigma_{\text {shadow }}$ and accounts for shadowing effects, $r_{j, i, k}$ is the distance between the $k^{\text {th }} \mathrm{UE}$ and the BS of the $j^{\text {th }}$ cell, $R$ is the cell radius, and $\alpha$ accounts for the path loss.

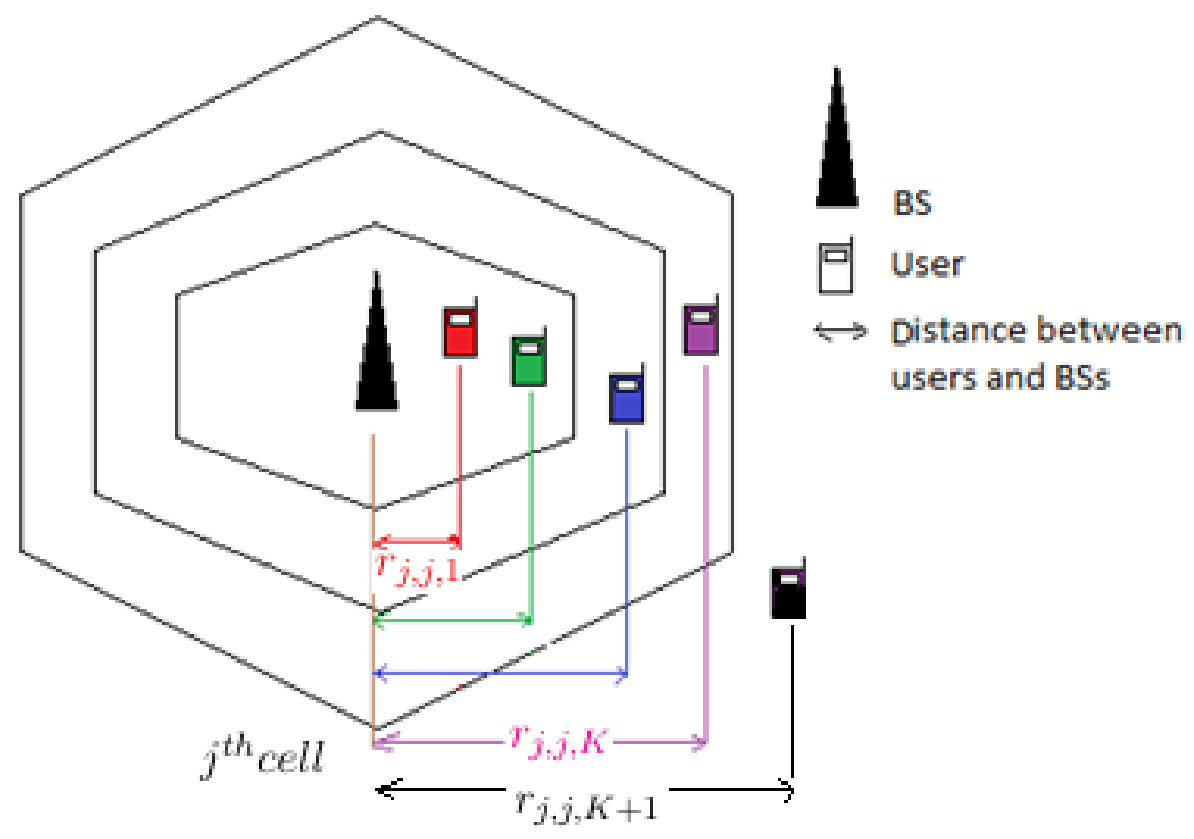

Figure 2. UEs' classification based on their $L S F^{\text {coef ficients }}$.

Therefore, from (11), the size of UEs' $L S F^{\text {coef ficients }}$ increases from the edge to the center of a cell; hence, we can exploit this fact to classify the UEs of each cell. Let consider the example depicted at Fig.2, where the BS of the $j^{t h}$ cell classifies its supported UEs in a matrix of classification labeled $\beta_{j}$, and the $(K+1)^{t h} \mathrm{UE}$ is considered out of the interest of the $j^{t h}$ BS. Hence, $\beta_{j}$ can be expressed as follows

$$
\beta_{j}=\left[\beta_{j, j, 1}^{2}>, \ldots,>\beta_{j, j, k}^{2}>, \ldots,>\beta_{j, j, K}^{2}\right]
$$

It is very important to remember that the number of UEs is the same in each cell (i.e., $K$ UE per cell). In the second step, all BSs send their constructed matrices of classification $\beta_{j}: j=1,2, \ldots, L$ to a network hub, which is charged to construct a global matrix of classification, which can be expressed as follows

$$
\beta=\left[\beta_{1}^{T}, \ldots, \beta_{j}^{T}, \ldots, \beta_{L}^{T}\right]^{T}
$$




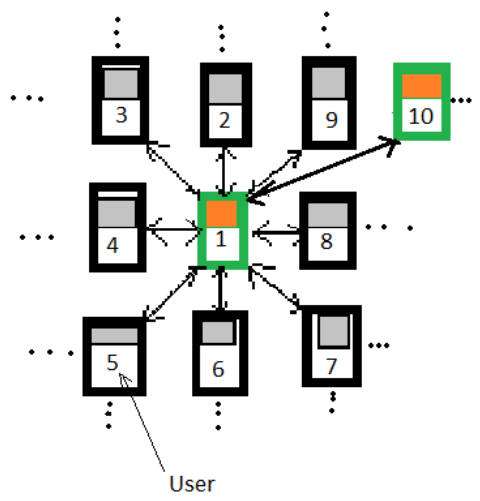

Figure 3. The UE indexed by one is assigned with a pilot $\phi^{[1]}$ and the proposed algorithm does not allow the reuse of the same pilot for the UEs 2,3...9, while it can be used for the UE 10

Based on the proof given in the appendix 9, the problem of PC diminishes when the distance between UEs that employ the same pilots is large. Accordingly, the proposed algorithm assigns different pilot sequences to the UEs which are close to each other, this through the following proposition

Proposition:

Let $P \in C^{(L \tau) \times K}$ be the pilot assignment matrix of the UEs of all cells (i.e L cell) and $S=\left[\phi^{[1]}, \phi^{[2]}, \ldots, \phi^{[K]}\right] \in$ $C^{\tau \times K}$ is a set of the available OPSs of length $\tau$, and it contains at least $K$ OPSs. Hence, the rows of P can be expressed for a cluster of $\mathrm{L}=7$ hexagonal cells as follows:

$$
\begin{aligned}
& P(i, k=1,2, \ldots, K)_{i=1,2, \ldots, L}= \\
& = \begin{cases}S & \text { ifi }=1 \text { or } \theta_{i}>K \\
\operatorname{fliplr}(S) & i=2 \\
{\left[S\left(1, \theta_{i}: \max (S)\right), S\left(1,1: \theta_{i}-1\right)\right]} & i>2 \text { and } \theta_{i} \leq K\end{cases}
\end{aligned}
$$

Where $\theta_{i}=2 i-3$, and fliplr(vector) is the rotation of a vector by $\pi\left(\right.$ e.g $\left.\operatorname{flipl}\left(\left[\begin{array}{lll}1 & 2 & 3\end{array}\right]\right)=\left[\begin{array}{lll}3 & 2 & 1\end{array}\right]\right)$.

Notice that in (14) and during the process of constructing the matrix $P$, it is possible to find that two pilots are assigned to two UEs, which are close to each other in the matrix P (e.g Fig.3, where we have considered $d_{\text {step }}=1$ ), leading in fact to generating severe contamination. Hence, to avoid this constraint, the proposed algorithm checks the following constraint:

For $\{i=(1,2, \ldots, 7), k=1, \ldots, K\}$, check if:

$$
\begin{gathered}
P(i, k)=\left\{P\left(i-d_{\text {step }}, k-d_{\text {step }}\right), P\left(i-d_{\text {step }}, k\right), P\left(i-d_{\text {step }}, k\right), P\left(i-d_{\text {step }}, k+d_{\text {step }}\right),\right. \\
\left.P\left(i+d_{\text {step }}, k+d_{\text {step }}\right), P\left(i+d_{\text {step }}, k\right), P\left(i+d_{\text {step }}, k+d_{\text {step }}\right)\right\}
\end{gathered}
$$

If (15) is satisfied once, then the pilot in $\mathrm{P}(\mathrm{i}, \mathrm{k})$ is replaced by an additional OPSs.

Once, the matrix P is constructed, the network hub sends the $i^{t h}$ row of $\mathrm{P}$ to the $i^{t h} \mathrm{BS}$ (and so on for all BSs of the same cluster), then each BS assigns the received vector of pilots to its supported UEs where the $k^{t h}$ UE having the $L S F^{\text {coefficient }} \beta_{j, j, k}$ is assigned with the pilot of the $k^{\text {th }}$ position in the received pilot assignment vector and so on for the rest of UEs as depicted in Fig. 4. The algorithm of UEs' classification and pilot assignment is summarized in Algorithm.1.

Algorithm 1 1: Input: System parameters $d_{\text {step }}, K, L, S, \mu \beta_{j, j, K} /\{j=1, \ldots, L: k=1,2, \ldots, K\}$. 


\begin{tabular}{|c|c|}
\hline Pilot sequences & $P(i,:)=\left[\phi^{[2]}, \phi^{[K]}, \ldots, \phi^{[3]}\right]$ \\
\cline { 1 - 1 } LSF coefficients & \multirow{2}{*}{$\beta_{i}=\left[\beta_{i . i, 1}, \beta_{i, i, K}, \ldots, \beta_{i, i, 3}\right]$} \\
\cline { 1 - 1 } Users & $\square \quad \square \ldots . \square$ \\
\hline
\end{tabular}

Figure 4. Pilot Assignment based on the received P from the network hub

2: Output: The matrix of pilot assignment $P$, the number of pilot requirement $S$.

3: Each BS constructs a vector of its corresponding $L S F^{\text {coefficients }}$ classified in descendent order (12).

4: BSs send their corresponding vectors $\beta_{j}$, for $j=1,2, \ldots, L$, to a network hub.

5: The network hub arranges the received vectors in a matrix of classification (13).

6: Based on (14) and (15), the network hub constructs the matrix of pilot assignment (i.e P).

7: For $j=1, \ldots, L$, the network hub sends the $j^{t h}$ row of P to the $j^{t h} \mathrm{BS}$.

8: BSs assign the received vectors to their supported UEs i.e $\left\{\beta_{j, j, k} \leftarrow P(j, k)\right\}$ for $\{j=1, \ldots, L: k=$ $1,2, \ldots, K\}$.

9: end.

Notice that if the number of the considered cells is greater than seven, then the $L$ cells are divided into clusters of seven cells and the same analysis presented above can be repeated for each cluster.

\subsection{Power control algorithm}

The UEs face different channel conditioning; hence, it is unfair to force the UEs to use the same power for transmuting their data and pilot sequences, likewise, it is unfair to force the BSs to use the same power for downlinking data symbols to the UEs. Hence, we propose to control the power in both sides (i.e., BSs and UEs side). For that, let back to (5)(6), from the two equations, we notice that their denominator depends on the power of transmission, which affect the quality of fulfilled SINR. For example, increasing $\rho_{u}^{k}$ in (5) leads to reduce the effect of the noise, which can improve the quality of the fulfilled SINR.

Since each BS is charged to support only $K$ UEs, and after classifying the UEs of each cell in (12). Let focus solely on the uplink phase; the UEs are allowed to use different power levels for transmitting their data symbols, specifically, we fix a range of $\mathrm{K}$ power levels, and the smallest power is allocated to the closest UE to the BS, and the power is increased from the center to the edge of each cell. To clarify the concept of this proposal, let consider a single cell system of $\mathrm{K}=3\left(U E_{1}, U E_{2}\right.$ and $\left.U E_{3}\right)$ randomly distributed UEs, and the range of power levels is $\rho=[51015] \mathrm{dBm}$. Let assume that the three UEs are classified as that $U E_{2}$ is the closest UE to the BS, while the $U E_{1}$ is the furthest one; accordingly, $U E_{1}$ is allowed to use $\rho=15 \mathrm{dBm}$, and $\rho=10 \mathrm{dBm}$ for $U E_{3}$, and $\rho=5 \mathrm{dBm}$ for $U E_{2}$.

\section{Simulation Results}

In this section, we evaluate the performance of the proposed algorithm through a set of Monte Carlo simulations. We adopt a low complexity MF and zero-forcing detector and precoders, respectively, for data detection and data precoding, where we adopt the multi-cell system presented above. The parameters used in our simulations are given in Table 1. Herein, we compare the performance of our proposed strategy with the soft pilot reuse (SPR) for 
two different detectors and precoders (i.e zero-forcing (ZF) and matched filter (MF)), these through the average achievable rate, which can be expressed as

$$
C_{j, k}=(1-\gamma) E\left\{\log _{2}\left(1+S I N R_{j, k}\right)\right\}
$$

Where $\gamma$ accounts for the loss of the SE, which is caused by the problem of the pilot overhead, and it can be expressed as

$$
\gamma= \begin{cases}K_{\text {spr }} * \mu / K & \text { For } S P R \text { scheme } \\ S * \mu / K & \text { For the proposed scheme }\end{cases}
$$

where $S$ refers to the number of the OPSs used in our proposed strategy, while $K_{s p r}$ is the number of the OPSs used in the SPR of [26]. The value of both $K_{s p r}$ and $S$ depends on the distribution of the UEs within cells; hence, they cannot be fixed.

Table 1. System settings

\begin{tabular}{|c|c|}
\hline Number of cells & $L=7$ \\
\hline Number of antennas' BS & $32 \leq M \leq 256$ \\
\hline Number of UEs per-cell & $K=10$ \\
\hline Number of pilots S & $K<S<<L K$ \\
\hline Cell radius & $R=500 \mathrm{~m}$ \\
\hline Inner radius & $r=30 \mathrm{~m}$ \\
\hline Log normal shadow fading & $\sigma_{\text {shadow }}=8 \mathrm{~dB}$ \\
\hline Transmit power during the uplink training $\rho_{p}$ & $10 \mathrm{dBm}$ \\
\hline Transmit powers $\rho_{u}$ and $\rho_{d}$ & $10 d B m \leq \rho_{u}, \rho_{d} \leq 28 \mathrm{dBm}$ \\
\hline Path loss exponent & $\alpha=3$ \\
\hline Pilot overhead parameter $\mu$ & $0<\mu<1$, fixed $\mu=0.1$ \\
\hline
\end{tabular}

Fig. 5 depicts the average uplink achievable rate versus the number of the antennas BSs. The two linear detectors MF and ZF are employed for comparing the performance of our proposed strategy to those of the SPR strategy. It is well seen that the proposed strategy outperforms the SPR strategy in both detectors; specifically, at $M=32$, our proposed strategy is better by about $5 \mathrm{bps} / \mathrm{Hz}$ than the SPR strategy for MF detector, and the gap increases by increasing M. Similarly, at $M=32$, the proposed strategy is about $7 \mathrm{bps} / \mathrm{Hz}$ better than the SPR in the case of ZF detector, and the gap increases to reach about $12 \mathrm{bps} / \mathrm{Hz}$ at $M=256$. This can be explained by the fact that increasing the number of the antennas' BSs leads to strengthening the transmitted signals toward the targeted UEs, which helps to enhance the quality of the wireless communication; furthermore, a large number of the antennas at the BSs helps to focus the transmitted data signals towards the UEs, which leads to reduce the compensate the harmful influence of interference that takes place within cells. These achievements were reached only with $d_{\text {step }}=1$, which means that our strategy dose not require the use of a large number of the OPSs to overcome the PC problem.

The average downlink achievable rate versus $\mathrm{M}$ is depicted in Fig. 6, where the $\mathrm{ZF}$ and MF precoders are used to compare the performance of the proposed strategy to those of the SPR strategy. Similarly to the uplink phase, the proposed strategy outperforms the SPR in both precoders by about $5 \mathrm{bps} / \mathrm{Hz}$ at $M=32$, and the gap increases by increasing $\mathrm{M}$ to reach about $9 \mathrm{bps} / \mathrm{Hz}$ for $\mathrm{MF}$ and $10 \mathrm{bps} / \mathrm{Hz}$ for the ZF precoder at $M=256$. Similarly to the discussion of the uplink phase, these achievements proves the quality of the proposed strategy to overcome the PC problem within multi-cell M-MIMO systems. 


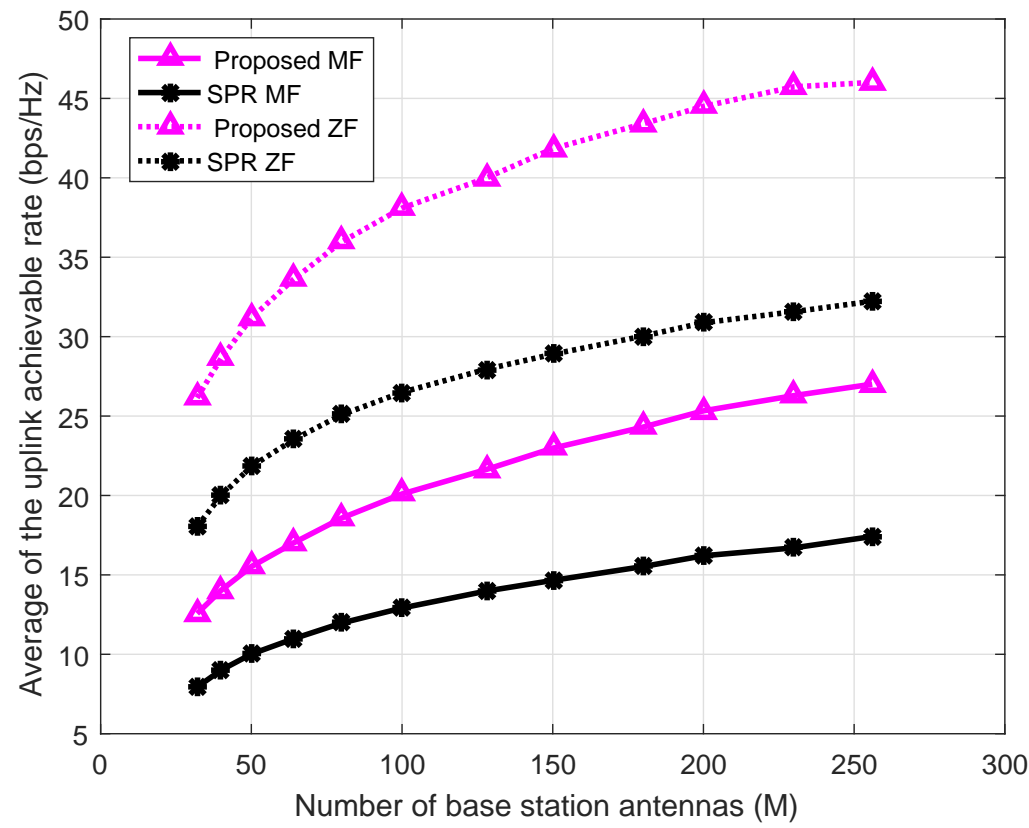

Figure 5. Average uplink achievable rate versus the number of antennas BSs, $d_{\text {step }}=1$

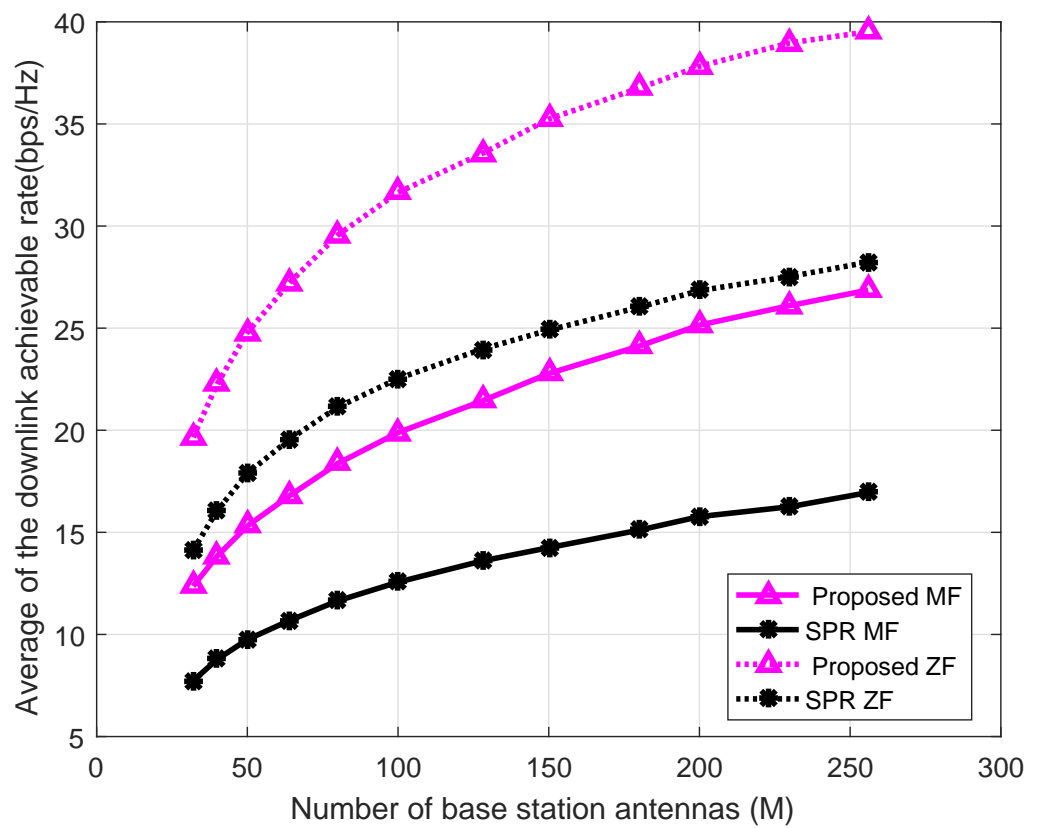

Figure 6. Average of the downlink achievable rate versus the number of antennas BSs, $d_{\text {step }}=1$. 


\section{Conclusion}

This paper proposes the consolidation of a pilot assignment scheme with a power control-based strategy, which proves that it can lead to enhance the quality-of-service for the overall UEs, instead of their geographical position, within cells. Specifically, the UEs' large-scale fading coefficients are exploited to classify the UEs within cells, and based on that, the UEs are assigned with their pilot sequences, which leads to reduce the influence of the PC problem. Since the UEs are subject to such a different channel conditions, we have allowed the edge UEs to use maximum power for transmitting their data symbols, and as closer be the UE to its supported BS, as lower it become the power that can he use for data transmission, and similarly in downlink phase. The effectiveness of our proposed strategy is proved through simulation results.

\section{Appendix}

Let consider the following expression of the $S I N R^{j, k}$ of a UE $k$ of the $j^{t h}$ cell:

$$
S I N R^{j, k}=\frac{\beta_{j, j, k}^{2}}{\sum_{i \neq j}^{L} \beta_{j, i, k}^{2}}
$$

Therefore the $L S F^{\text {coef ficient }}$ of the $k^{t h}$ UEs of the $i^{t h}$ cell to the BS of the $j^{t h}$ cell is modulated according to [3] as

$$
\beta_{j, i, k}=\frac{z_{j, i, k}}{\left(r_{j, i, k} / R\right)^{\alpha}}
$$

thereafter, we replace the $L S F^{\text {coef ficient }}$ in (18) based on (19) as follows:

$$
S I N R^{j, k}=\frac{\frac{z_{j, j, k}^{2}}{\left(r_{j, j, k} / R\right)^{2 \alpha}}}{\sum_{i \neq j}^{L} \frac{z_{j, i, k}}{\left(r_{j, i, k} / R\right)^{2 \alpha}}}=z_{j, j, k}^{2} \frac{1}{\sum_{i \neq j}^{L} z_{j, i, k}^{2}\left(\frac{r_{j, j, k}}{r_{j, i, k}}\right)^{2 \alpha}}
$$

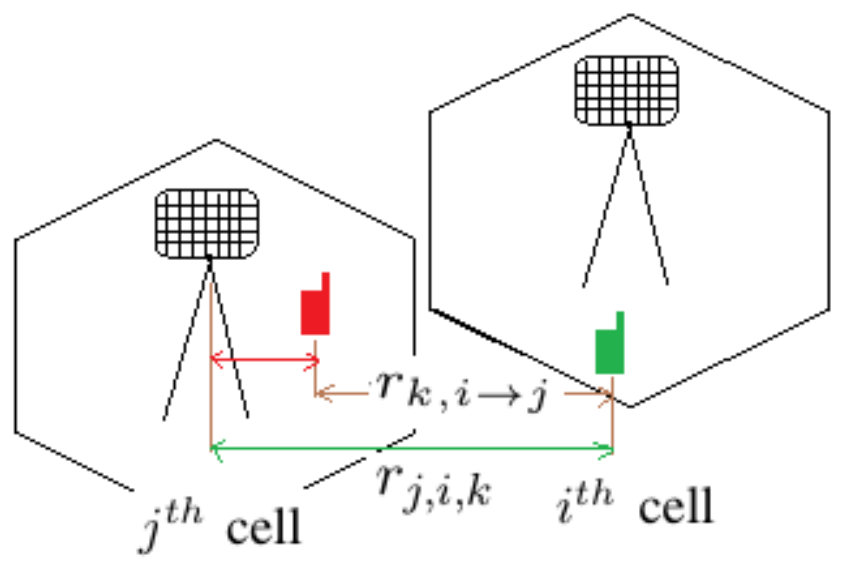

Figure 7. Distance between two UEs -of different cells- that employ the same pilot

Based on Fig. 7, we have: $r_{j, i, k}=r_{k, i \rightarrow j}+r_{j, j, k}$, therefore (20) becomes:

$$
S I N R^{j, k}=z_{j, j, k}^{2} \frac{1}{\sum_{i \neq j}^{L} z_{j, i, k}^{2}\left(\frac{r_{j, j, k}}{r_{j, j, k}+r_{k, i \rightarrow j}}\right)^{2 \alpha}}
$$

Then, based on the denominator of (20), as the distance $r_{k, i \rightarrow j}$ (i.e between two UEs of different cells having the same pilot sequence Fig.7) increases the quantity $\frac{r_{j, j, k}}{r_{j, j, k}+r_{k, i \rightarrow j}}$ decreases, therefore the $S I N R^{j, k}$ increases, which 
lead to reach an improved SINR of the considered UEs, owing from this expression the proposed algorithm assigns pilot to UEs with the aim of increasing the distance between UEs that employ the same pilots.

\section{REFERENCES}

1. 12th annual Mobile VNI Forecast. Explore global and regional mobile data traffic growth and trends, (2017-2022)

2. E. G. Larsson, O. Edfors, F. Tufvesson, T. L. Marzetta, Massive MIMO for Next Generation Wireless Systems, IEEE Communications Magazine, 52(2), 186-195, (2014)

3. T. L. Marzetta, Noncooperative Cellular Wireless with Unlimited Numbers of Base Station Antennas, IEEE Transactions on Wireless Communications, vol. 9, no. 11, pp. 3590-3600, November 2010.

4. A. Belhabib, M. Boulouird and M. M. Hassani , The Impact of Using Additional Pilots on the Performance of Massive MIMO Systems, IEEE International Conference on Signal, Control and Communication (SCC 2019), 16-18 December 2019, Hammamet, Tunisie

5. J. Fan, W. Li and Y. Zhang, Pilot contamination mitigation by fractional pilot reuse with threshold optimization in massive MIMO systems, Digital Signal Processing, Vol. 78, Pages. 197-204, 2018.

6. H. Q. Ngo, M. Matthaiou and E. G. Larsson, Massive MIMO With Optimal Power and Training Duration Allocation, IEEE Wireless Communications Letters, vol. 3, no. 6, pp. 605-608, Dec. 2014.

7. L. Hao, W. Zhigang and W. Houjun, An energy-efficient power allocation scheme for Massive MIMO systems with imperfect CSI, Digital Signal Processing, 102964, ISSN 1051-2004, 2021.

8. L. Shao, Coordinated multicell beamforming and power allocation for massive MIMO: A large system analysis, Signal Processing, Volume 164, 2019, Pages 41-47, ISSN 0165-1684.

9. H. Dahrouj, W. Yu, Coordinated beamforming for the multicell multi-antenna wireless system, IEEE Trans. Wirel. Commun. 9 (5), $1748-1759,2010$.

10. A. Tolli, H. Pennanen and P. Komulainen, Decentralized Minimum Power Multi-Cell Beamforming with Limited Backhaul Signaling, IEEE Transactions on Wireless Communications, vol. 10, no. 2, pp. 570-580, February 2011.

11. A. Ashikhmin, L. Li and T. L. Marzetta, Interference Reduction in Multi-Cell Massive MIMO Systems With Large-Scale Fading Precoding, IEEE Transactions on Information Theory, vol. 64, no. 9, pp. 6340-6361, Sept. 2018.

12. B. Hassibi and B. M. Hochwald, How much training is needed in multiple-antenna wireless links?, IEEE Transactions on Information Theory, vol. 49, no. 4, pp. 951-963, April 2003.

13. L. Li, A. Ashikhmin, and T. L. Marzetta, Interference reduction in multi-cell massive MIMO Systems II: Downlink analysis for a finite number of antennas, [Preprint], arXiv:1411.4183, 2014.

14. X. Zhu, L. Dai and Z. Wang, Graph Coloring Based Pilot Allocation to Mitigate Pilot Contamination for Multi-Cell Massive MIMO Systems, IEEE Communications Letters, vol. 19, no. 10, pp. 1842-1845, Oct. 2015.

15. F. Fernandes, A. Ashikhmin and T. L. Marzetta, Inter-Cell Interference in Noncooperative TDD Large Scale Antenna Systems, IEEE Journal on Selected Areas in Communications, vol. 31, no. 2, pp. 192-201, February 2013.

16. J. Zhang, B. Zhang, S. Chen, X. Mu, M. El-Hajjar and L. Hanzo, Pilot Contamination Elimination for Large-Scale Multiple-Antenna Aided OFDM Systems, IEEE Journal of Selected Topics in Signal Processing, vol. 8, no. 5, pp. 759-772, Oct. 2014.

17. Y. Wu, T. Liu, M. Cao, L. Li, W. Xu, Pilot contamination reduction in massive MIMO systems based on pilot scheduling, J Wireless Com Network, 21, 2018.

18. J. C. M. Filho, C. M. Panazio,T. Abrão, Joint uplink and downlink optimization of pilot assignment for massive MIMO with power control, Trans Emerging Tel Tech. 2017; 28:e3250.

19. S. M. Sanaie, H. K. Bizaki, Performance analysis of multicell massive MIMO THP with pilot contamination, Trans Emerging Tel Tech. 2019; 30:e3574.

20. T. Lee, H. Kim, S. Park and S. Bahk, Mitigation of sounding pilot contamination in massive MIMO systems, 2014 IEEE International Conference on Communications (ICC), Sydney, NSW, 2014, pp. 1191-1196.

21. Z. Wang, P. Zhao, C. Qian and S. Chen, Location-Aware Channel Estimation Enhanced TDD Based Massive MIMO, IEEE Access, vol. 4, pp. 7828-7840, 2016.

22. X. Zhu, Z. Wang, L. Dai and C. Qian, Smart Pilot Assignment for Massive MIMO, IEEE Communications Letters, vol. 19, no. 9, pp. 1644-1647, Sept. 2015.

23. O. Elijah, C. Y. Leow, T. A. Rahman, S. Nunoo and S. Z. Iliya, A Comprehensive Survey of Pilot Contamination in Massive MIMO-5G System, IEEE Communications Surveys \& Tutorials, vol. 18, no. 2, pp. 905-923, Secondquarter 2016.

24. X. Zhu, L. Dai, Z. Wang and X. Wang, Weighted-Graph-Coloring-Based Pilot Decontamination for Multicell Massive MIMO Systems, IEEE Transactions on Vehicular Technology, vol. 66, no. 3, pp. 2829-2834, March 2017.

25. A. Belhabib, M. Boulouird, M. M. Rabet Hassani, Smart Switching Strategy-Based Supervision Rule to Mitigate the Problem of Pilot Contamination in Massive MIMO Systems, Wireless Communications and Mobile Computing, vol. 2020, Article ID 8868597, 13 pages, 2020.

26. X. Zhu, Z. Wang, C. Qian, L. Dai, J. Chen, S. Chen and L. Hanzo, Soft Pilot Reuse and Multicell Block Diagonalization Precoding for Massive MIMO Systems, IEEE Transactions on Vehicular Technology, vol. 65, no. 5, pp. 3285-3298, May 2016. 